\title{
Mythos
}

Rivista di Storia delle Religioni

12 | 2018

Varia

\section{Gerechte Götter? Vorstellungen von göttlicher Vergeltung im Mythos und Kult des archaischen und klassischen Griechenlands}

(“Universitätsbibliothek") Heidelberg, Propylaeum, pp. 358, ISBN 972-3-946654-52-0,€ 24,90

\section{Gian Franco Chiai}

\section{OpenEdition}

\section{Journals}

Edizione digitale

URL: http://journals.openedition.org/mythos/357

DOI: $10.4000 /$ mythos.357

ISSN: 2037-7746

\section{Editore}

Salvatore Sciascia Editore

\section{Edizione cartacea}

Data di pubblicazione: 1 dicembre 2018

Paginazione: 142-144

ISBN: 978-88-8241-501-3

ISSN: $1972-2516$

\section{Notizia bibliografica digitale}

Gian Franco Chiai, « Gerechte Götter? Vorstellungen von göttlicher Vergeltung im Mythos und Kult des archaischen und klassischen Griechenlands », Mythos [Online], 12 | 2018, online dal 24 septembre 2019, consultato il 24 septembre 2020. URL : http://journals.openedition.org/mythos/357 ; DOI : https://doi.org/10.4000/mythos.357 


\section{Irene Berti}

Gerechte Götter? Vorstellungen von göttlicher Vergeltung im Mythos und Kult des archaischen und klassischen Griechenlands

(“Universitätsbibliothek”) Heidelberg, Propylaeum, pp. 358, ISBN 972-3-946654-52-0, € 24,90.

\section{Gian Franco Chiai - Freie Universität Berlin - gian.franco.chiai@fu-berlin.de}

II libro qui recensito rappresenta la versione rivista e bibliograficamente aggiornata di una tesi di dottorato discussa ad Heidelberg nel 2007 e scritta sotto la direzione del prof. A. Chaniotis. La monografia, pubblicata on-line come e-book nella serie Propylaeum della "Universitätsbibliothek" di Heidelberg, costituisce a parere del recensore un lavoro, stimolante e ricco di spunti che possono essere approfonditi. Per tal motivo vi è da augurarsi che i risultati di questo lavoro vengano recepiti e proficuamente messi a frutto nel mondo accademico. Lo spazio ristretto di una recensione non permette di discutere in maniera approfondita tutti i temi trattati dall'autrice. Per tal motivo, dopo aver esposto e riassunto la struttura ed il contenuto dei singoli capitoli, mi soffermerò con alcune note critiche su alcuni aspetti che hanno maggiormente attirato la mia attenzione.

La monografia si articola in 12 capitoli, suddivisi in tre parti: parte A (cap. I-III), parte B (cap. IV-X) e parte C (cap. XI-XII). II libro si apre con un'ampia introduzione (pp. 1328), nella quale si discute il significato del termine moderno Gerechtigkeit, una parola tedesca il cui campo semantico non trova una corrispondenza esatta nei termini greci dike, themis, dikaiosyne etc. La stessa difficoltà si riscontra per altro anche in italiano, in cui per rendere appieno il significato di questo concetto si è costretti a ricorrere a perifrasi come "senso di giustizia", "quello che è giusto" etc. Questa introduzione mostra acutamente la difficoltà nel trovare un equivalente esatto per idee e concetti moderni nelle lingue e nelle culture antiche. Le stesse considerazioni, come giustamente l'autrice sottolinea, possono essere fatte viceversa anche in relazione al greco antico, in cui il concetto di dike può coprire il campo semantico di Rache (vendetta), Vergeltung (ritorsione) e Gerechtigkeit (senso di giustizia).

II primo capitolo (pp. 29-51) si sofferma a studiare i meccanismi dell'intervento divino, finalizzato a ristabilire la giustizia e a punire quanti con le loro azioni hanno trasgredito norme etiche e sociali, facendo un torto a qualcuno. In questo senso la Gerechtigkeit antica si lascia interpretare come espressione di un processo di reciprocità. II secondo capitolo (pp. 53-71) è dedicato all'analisi della colpa, che produce impurità. A riguardo va segnalata l'utile analisi della terminologia antica (agos, enages, miasma etc.), ben illustrata attraverso lo studio di esempi concreti tratti da opere letterarie. II terzo capitolo (pp. 73-87) rappresenta a mio avviso uno dei più originali e meglio riusciti della monografia. Qui vengono studiati i media della comunicazione religiosa usati per prendere contatto con una divinità e spesso con toni patetici convincerla ad intervenire, ai fini non solo di ristabilire la giustizia, ma anche di punire il malfattore, dando soddisfazione al senso di vendetta e di rivalsa di chi aveva (o meglio avrebbe) subito il torto. A riguardo l'autrice, mostrando una profonda conoscenza dei testi epigrafici, prende in considerazione la categoria delle c.d. "preghiere per la giustizia" (prayers for justice - si tratta di un termine coniato dallo studioso olandese $\mathrm{H}$. Versnel). A tal fine vengono studiate le iscrizioni del santuario di Demetra e Kore a Cnido, apposte su tavolette plumbee e affisse poi sulle pareti del tempio. Queste testimonianze epigrafiche vengono analizzate e comparate con altri famosi testi, come ad esempio la Sakrileg-Inschrift del tempio di Alea a Mantinea. La comparazione tra testimonianze letterarie ed epigrafiche conduce a nuove ed 
interessanti conclusioni, come ad esempio quella del constatare che la connessione tra malattia e mancanza - ciò che J. Assmann nei suoi scritti riferendosi al mondo egizio ed ebraico ha definito come konnektive Gerechtigkeit - è attestata non solo in epoca romana, ma trova interessanti testimonianze presso i Greci già in epoca arcaica nei poemi omerici.

La seconda parte, che inizia con una sintetica introduzione al tema (cap. IV, pp. 89-95) analizza singolarmente le diverse figure divine legate alla sfera della giustizia (Gerechtigkeit). II cap. V (pp. 97-137) tratta delle Semnai Theai, delle Eumenidi e delle Erinni - denominazioni diverse indicanti la medesima divinità che assolve funzioni differenti - analizzando le caratteristiche rituali del loro culto nei contesti locali ad Atene ed in Attica. II cap. VI (pp. 139-176) si incentra sulle Eumenidi e sui rispettivi luoghi di culto nel Peloponneso (Argo, Sicione, Megalopoli etc.) e nelle colonie doriche d'oltremare (Cirene, Selinunte). Interessante ed originale è lo studio comparato della lex sacra di Selinunte e delle iscrizioni cirenaiche. II cap. VII (pp. 177-227) è dedicato alle Erinni ed alla diffusione del loro culto nel mondo greco (Arcadia, Beozia etc.); di particolare rilievo sono a tal proposito le connessioni osservate tra le Erinni e la fertilità, evidenti nel culto di Demetra Erinni a Telpusa (pp. 200-201); di utilità è pure la raccolta e la puntuale analisi delle fonti letterarie e documentarie (papiro di Derveni, defixiones etc.) in cui queste divinità vengono menzionate. II cap. VIII (pp. 229-262) offre una dettagliata analisi di Zeus, quale istanza più alta della giustizia divina; l'autrice studia gli epiteti cultuali e le connessioni del re dell'Olimpo con le altre divinità connesse alla Gerechtigkeit. Successivamente vengono prese in esame le figure dei Daimones Alastores con un'utile raccolta ed analisi della documentazione attinente. II cap. IX (pp. 263-280) è dedicato allo studio del culto delle Praxidikai e di Dike, mentre il cap. X (pp. 281-309) tratta di Nemesi e dei suoi luoghi di culto (Ramnunte, Smirne etc.).
II chiaro pregio di questa parte consiste nell'ampia raccolta e discussione di un impressionante numero di testimonianze letterarie, epigrafiche ed archeologiche relative al culto di tutte queste divinità, che, prescindendo da singoli studi, non erano ancora state trattate in maniera sistematica e in modo comparativo in una monografia. Proprio in questa parte si evince uno dei punti di forza di questo lavoro: I'interdisciplinarietà, che permette all'autrice di utilizzare dati archeologici, epigrafici e testimonianze letterarie per ricostruire le caratteristiche di queste divinità della giustizia, partendo dai contesti materiali dei loro luoghi di culto.

La terza parte, comprendente i cap. XIXII (pp. 311-317, 318-324), tematizza il ruolo della Gerechtigkeit nelle pratiche cultuali $\mathrm{e}$ in generale nel discorso culturale della Grecia arcaica e classica, definendo la morfologia di questi esseri divini, spesso adorati in gruppi dal numero non ben definito, quali onniscienti e connessi alla fertilità, e ricostruendo il loro sviluppo nel corso dei secoli. Paradigmatico è ad esempio il caso del culto delle Erinni, il cui culto sopravviveva in Arcadia ancora all'epoca di Pausania, le cui caratteristiche negative sembrano rafforzarsi in epoca imperiale, come mostrano le loro numerose menzioni nelle defixiones. Basandosi sulle fonti letterarie e documentarie, l'autrice sottolinea nel complesso il forte carattere locale e spesso legato alla sfera privata del culto di queste divinità.

La lettura di quest'opera offre, come accennato, anche diversi spunti critici e di approfondimento. Eccone qui due, relativi rispettivamente al concetto di teodicea ed alla concorrenza tra giustizia divina e giustizia secolare. La teodicea (dal greco theos, "dio", e dike, "giustizia") è quella branca della teologia e della storia delle religioni che indaga il problema di come si concili l'esistenza di un dio onnipotente, buono e giusto con la presenza del male nell'esistenza umana. Questo termine, benché derivato dal greco antico, non risulta essere attestato presso gli autori classici, i quali erano per altro consci di questa problematica (l'autrice 
rimanda a riguardo all'informativo lavoro di S. Lanzi, THEOS ANAITIOS. Storia della teodicea da Omero ad Agostino, Roma 2000). II termine teodicea è attestato per la prima volta nell'opera di G.W. Leibniz (Essais de théodicée, I), pubblicata ad Amsterdam nel 1710. II filosofo utilizzava questo termine in relazione alla giustificazione dell'esistenza e del ruolo di Dio (qui in riferimento al Dio cristiano) per il male presente nel mondo: egli attribuiva l'esistenza del male e delle sofferenze alla libertà umana del libero arbitrio, concessa all'uomo da Dio.

Compresa l'autrice, molti studiosi moderni (Versnel, Chaniotis etc.) usano tuttavia questo termine in un'accezione più ampia, riferendosi al modo in cui una divinità interviene nelle faccende umane in difesa dei suoi fedeli, ristabilendo a loro vantaggio la giustizia e facendo in modo che le norme etiche e sociali vengano ristabilite e rispettate. Si tratta di un'importante precisazione che sarebbe stato opportuno, a mio avviso, fare nel libro.

Un altro aspetto che, a mio parere, si sarebbe potuto approfondire attiene al rapporto tra pratiche cultuali legate alla giustizia ed il loro rapporto con la giustizia secolare. Le testimonianze prese in esame permettono infatti di ricostruire uno scenario di velata concorrenza tra istituzioni religiose ed autorità giudiziarie. Come l'autrice giustamente fa notare, non è un caso che la maggior parte dei testi provenienti dal santuario di Demetra e di Kore di Cnido e di Corinto siano da ricondurre a delle donne, probabilmente di ceto medio basso. Si tratta di una categoria sociale particolarmente debole, che non solo in epoca classica, ma anche nella più tarda epoca romana - non a caso, seguen- do l'esempio di H. Versnel e di A. Chaniotis, l'autrice considera le iscrizioni confessionali della Lidia e della Frigia - non era particolarmente tutelata. Testimonianze di epoca tardo ellenistica e romana - si potrebbero considerare un ostrakon dall'Egitto romano, contente la supplica di due fratelli contro le angherie di un signorotto locale (SB, XVIII, 13931), o le numerose preghiere per la giustizia rinvenute nel santuario di Bath, in Inghilterra, anche questo dedicato ad una divinità femminile identificata con Minerva - mostrano in maniera evidente come per molte persone, appartenenti ai ceti più bassi, la giustizia divina, amministrata da divinità infallibili ed onniscienti, rappresentasse una valida alternativa a quella umana, controllata da autorità spesso corruttibili, che non avevano a cuore il bene di un povero contadino e che stavano spesso dalla parte dei potenti. In questo senso possiamo rintracciare un filo rosso di continuità tra l'epoca arcaica e classica, trattata da I. Berti, e quella dei periodi ellenistico e romano. Si potrebbe anche aggiungere che la paura nei confronti di una punizione divina, che prima o poi si sarebbe abbattuta su di un colpevole e sulla sua famiglia - chi poteva infatti augurarsi di venire perseguitato giorno e notte dalle Erinni, come Oreste? - doveva giocare anche in quest'epoca un ruolo importante: questa paura, proprio come nel caso delle iscrizioni confessionali, doveva favorire il rispetto delle norme etiche sociali ed il quieto vivere.

Prescindendo da queste mie due note critiche, il libro di I. Berti rappresenta una lettura stimolante che indubbiamente arricchisce il lettore, fornendo, come detto, stimoli per ulteriori ricerche ed approfondimenti. 